\title{
Politización y CONFIANZA EN LOS MEdios de COMUNiCACión: Argentina durante EL KIRCHNERISMO
}

Politicization and Trust in the Media: Argentina during the Kirchner Years

\section{GERMÁN LODOLA}

Universidad Torcuato Di Tella, Argentina - CONICET

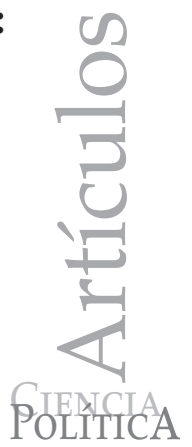

\section{PHILIP KITZBERGER}

Universidad Torcuato Di Tella, Argentina - CONICET

\begin{abstract}
RESUMEN
¿En qué medida la politización del orden mediático activada por gobiernos de "nueva izquierda" en América Latina, afectó la confianza ciudadana en los medios de comunicación? Utilizando datos a nivel individual en Argentina, donde el conflicto gobierno-medios fue intenso, este trabajo examina el impacto de tres variables políticas: preferencias, sofisticación y temas. La evidencia indica que los simpatizantes del kirchnerismo confían menos que las demás personas, mientras que los votantes evalúan los medios según el desempeño presidencial. Por otra parte, los individuos políticamente sofisticados expresan menores niveles de confianza, mientras que las víctimas de la delincuencia manifiestan mayores. Estos resultados tienen implicancias para el estudio de la política de medios y el papel de los encuadres de las elites sobre las percepciones individuales acerca de la prensa.
\end{abstract}

Palabras clave: medios, opinión pública, argentina, kirchnerismo, polarización

\begin{abstract}
To what extent did media politicization linked to "new left" governments in Latin America affect media trust? Using individual level data in Argentina, where the government-multimedia conflict was intense, we examine the impact of three political variables: sophistication, preferences, and issues. We find that persons who identified as kirchneristas express less trust, while government voters evaluate media outlets considering presidential performance. In contrast to public opinion in the US, conservatives reveal higher levels of trust. Moreover, individual attributes linked to political sophistication are negatively correlated with media trust, while victims of crime manifest lower levels of credibility. These results have implications for the study of media politics, and the role of elites' frames and rhetoric in shaping individual perceptions about mass media.
\end{abstract}

Key words: media, public opinion, Argentina, kirchnernismo, polarization 


\section{INTRODUCCIÓN}

En el contexto del ascenso al gobierno de la "nueva izquierda" en América Latina (Levitsky y Roberts 2011), la cuestión mediática ha tenido lugar un proceso de politización sin precedentes. Aunque con variaciones significativas, tanto los gobiernos de matriz populista como los tributarios de una izquierda partidaria cuestionaron la neutralidad de los grandes medios de comunicación, los que pasaron a ser encuadrados como actores con intereses políticos vinculados a poderosas corporaciones y sectores del establishment. En algunos casos, estos gobiernos incluso avanzaron reformas orientadas a regular el sistema de medios y alterar las relaciones de poder en el campo mediático.

La politización del orden mediático en América Latina no fue consecuencia de demandas preexistentes ni el resultado de la acción organizada de la sociedad civil. Aunque varios países experimentaron demandas por la democratización del sistema de medios, impulsadas por pequeñas redes de activistas, el cuestionamiento público y las reformas en el área solo ocurrieron donde medió la oportunidad política ligada a gobiernos que enfrentaron públicamente a los conglomerados mediáticos (Waisbord 2013; Vommaro y Schuliaquer 2014; Segura y Waisbord 2016; Kitzberger 2016). Así, la organización institucional del sector y el funcionamiento de las empresas de medios en las democracias latinoamericanas eran cuestiones ajenas a la opinión pública, las que fueron políticamente canalizadas "desde arriba" por gobiernos de nueva izquierda.

Una extensa tradición de estudios ha demostrado que las elites políticas son capaces de modelar las percepciones de la opinión pública a través de la utilización de "señales" (Converse 1964; Zaller 1992; Matsubayashi 2013), como la identificación partidaria (Campbell et al. 1960; Lewis-Beck et al. 2008; Nicholson 2009), y "encuadres" o marcos interpretativos basados en argumentaciones e historias emocionales (Bullock 2011). En línea con esta preocupación, este trabajo examina en qué medida, la práctica sistemática de los gobiernos de nueva izquierda de denunciar públicamente los sesgos políticos de los conglomerados mediáticos, afectó la confianza de la opinión pública en los medios de comunicación.

Este fenómeno es analizado a nivel individual en Argentina, donde el conflicto entre el gobierno y los grandes medios alcanzó un alto nivel de politización. Esto incluyó la construcción de un discurso público radicalmente crítico, el despliegue de dispositivos mediáticos controlados por el gobierno y la aprobación de una ambiciosa legislación reformista. Para explorar en qué medida este conflicto explica la variación observada entre los argentinos en la confianza en los medios, se utilizan datos de opinión pública generados por las rondas 2008, 2010, 2012 y 2014 del Barómetro de las Américas (Latin American Public Opinion Project, LAPOP). Afortunadamente, la primera ronda fue administrada antes de que estallara la disputa entre el gobierno kirchnerista y las empresas de 
medios a raíz de la sesgada cobertura de un lock out agropecuario ${ }^{1}$. Las rondas restantes fueron conducidas bastante tiempo después, una vez activados los dispositivos comunicacionales y la retórica denunciante del gobierno. Esta condición permite eliminar el sesgo partidario de las percepciones individuales sobre los medios y "exogeneizar" el efecto causal de los encuadres emitidos por las elites de gobierno.

La evidencia empírica efectivamente demuestra que estos marcos interpretativos y dispositivos mediáticos impactaron en un segmento considerable de la población. Los modelos de regresión muestran que, previo a la emergencia del conflicto, no existe una asociación estadísticamente significativa entre apoyar políticamente al kirchnerismo y expresar menores niveles de confianza en los medios. En cambio, los mismos modelos, estimados para el periodo posterior, sustancian que los simpatizantes políticos del kirchnerismo confían sistemáticamente menos que el resto de las personas, mientras que el nivel de confianza de los votantes es condicional a la evaluación del desempeño presidencial, a mayor/menor evaluación menor/mayor confianza en los medios.

Por otra parte, a diferencia de lo que ocurre en Estados Unidos, los resultados indican que las personas identificadas con ideologías conservadoras expresan mayores - y no menores- niveles de confianza. Este fenómeno está ligado al hecho de que, en Argentina, el periodismo tiene un bajo grado de independencia profesional respecto de los dueños de medios, típicamente vinculados a sectores de élite. Además, la evidencia muestra que, aun controlando por la confianza en otras instituciones, ciertos atributos individuales señalados por la tesis de la "sofisticación política" (el interés por las cuestiones públicas, el consumo de fuentes alternativas de información y la educación) están negativamente relacionados con la credibilidad en los medios. Por último, los datos señalan que las percepciones sobre la inseguridad y la corrupción no tienen efecto sobre el nivel de confianza, mientras que las víctimas del crimen - pero no las víctimas de la corrupción-confían menos que las no-víctimas.

Evaluar los determinantes de la confianza en los medios es relevante por varios motivos. Primero, la vitalidad de una democracia representativa descansa, en parte, sobre ciudadanos informados acerca de las cuestiones públicas. Segundo, esta evaluación permite identificar qué sectores de la población son más susceptibles a la influencia mediática y cuáles a los encuadres de las élites. Por último, la cuestión de la credibilidad de los medios es importante en otros países de América Latina, como Ecuador y Venezuela, donde el cuestionamiento público a las empresas e instituciones mediáticas se ha replicado con intensidad.

El resto del trabajo se organiza del siguiente modo. La segunda sección presenta el fenómeno de la politización del orden mediático en Argentina y describe la 
dinámica que asumió este proceso durante los gobiernos de Néstor Kirchner (2003-2007) y Cristina Fernández de Kirchner (CFK, 2007-2015). La tercera discute la literatura sobre confianza en los medios y elabora hipótesis empíricamente demostrables, que enfatizan el impacto de factores individuales de naturaleza política. La cuarta operacionaliza las variables de interés. La quinta evalúa las hipótesis mediante un análisis de regresión multivariado corregido por el efecto del diseño de la muestra. La sección final menciona las implicancias de este análisis para el estudio de la política de medios y sugiere algunas líneas de investigación futura.

\section{LA POLITIZACIÓN DE LOS MEDIOS DE COMUNICACIÓN}

El giro hacia la izquierda en América Latina colocó a los medios de comunicación en el centro de la escena política. Los gobiernos de esta extracción ideológica desplegaron, con variable radicalidad, mecanismos discursivos y legales orientados a cuestionar la neutralidad política de los grandes medios y buscaron modificar las relaciones de poder en el sector (Kitzberger 2012; Waisbord 2013). Esquemáticamente, este activismo mediático gubernamental presenta tres dimensiones.

La primera dimensión concierne a la elaboración de un discurso público crítico sobre medios y periodismo. El argumento es que los grandes medios son (o responden a) sectores del establishment y poderosas corporaciones. De esto se desprende que el poder en la esfera mediática reproduce las asimetrías sociales existentes, lo que convierte a los denominados "medios hegemónicos o dominantes" - grandes empresas periodísticas que controlan una porción significativa del mercado de noticias- en defensores del statu quo frente a las agendas democratizadoras de los gobiernos progresistas.

La segunda dimensión corresponde a la comunicación gubernamental y la relación con el periodismo. En general, los gobiernos de izquierda populista diseñaron un repertorio de prácticas orientadas a la comunicación directa con el público. Por un lado, construyeron dispositivos comunicacionales anclados en la figura presidencial: programas radiotelevisivos conducidos por el Presidente, uso de cadenas nacionales, gabinetes itinerantes y redes sociales. Por otro lado, delegaron ciertas iniciativas comunicacionales en sectores aliados dentro del periodismo y el campo comunicativo, las cuales tomaron la forma de programas y espacios dedicados a responder la agenda comunicacional opositora construida por los grandes medios. Ambas estrategias comportan el rechazo a los formatos convencionales del periodismo: la entrevista, la conferencia de prensa y los contactos regulares fuente-periodista.

Finalmente, la tercera dimensión refiere a los cambios introducidos en el sector mediático con el objetivo de promover lógicas alternativas al mercado, contrarias al poder de los medios tradicionales. Las iniciativas de reforma del marco regulatorio del sector son la principal expresión de esta estrategia. 
A continuación, se utilizan estas tres dimensiones para discutir los rasgos salientes de la relación entre el gobierno y los conglomerados de medios durante el kirchnerismo en Argentina.

\section{Activismo mediático gubernamental en Argentina}

La relación política entre el kirchnerismo y los grandes medios puede ser dividida en dos etapas. La primera corresponde a la presidencia de Néstor Kirchner y se caracteriza por una ambivalente convivencia en la que coexistieron ciertas confrontaciones discursivas y prácticas comunicacionales que evitaron la intermediación del periodismo, junto con decisiones políticas que fortalecieron la posición privilegiada de los conglomerados de medios en el mercado. La segunda etapa tuvo lugar durante las presidencias de CFK y se destaca por una radicalización del discurso y las prácticas comunicacionales, junto a la reforma del marco regulatorio del sistema mediático (Kitzberger 2016).

Néstor Kirchner accedió a la presidencia con apenas el 22\% de los votos, el Partido Justicialista dividido y un nivel considerable de descontento social con la clase política tras la crisis de 2001. El presidente asumió con la promesa de renovar la política y el peronismo. Para ello, debía apelar a sectores medios urbanos no peronistas fuertemente expuestos a la influencia de los medios en la construcción de sus percepciones políticas. Así, la demanda a los grandes medios cobró enorme centralidad en la estrategia refundacional del kirchnerismo (Sivak 2015; Kitzberger 2016).

Durante el gobierno de Néstor Kirchner, la crítica pública a los medios periodísticos fue relativamente intensa. De los 862 discursos presidenciales, un cuarto contiene alusiones negativas a los medios (Vincent 2014). El discurso kirchnerista desplegó claves ideológicas que presentaban a los medios dominantes como agentes protagónicos de la "hegemonía neoliberal" de los años noventa, al naturalizar las visiones tecnocráticas de los "poderes fácticos" (no electivos) en detrimento de la política. Sin la centralidad que tendría luego, el gobierno ya postulaba, en esta etapa, el carácter engañoso del "relato mediático". En palabras de Néstor Kirchner: "nos quieren escribir el país a través de su diario o de los medios. Se tienen que dar cuenta que los argentinos ya hemos aprendido a desconfiar de ciertas cosas" (Vincent 2014: 373).

En cuanto a la comunicación gubernamental, la segunda dimensión de análisis, debe recordarse que Kirchner - gobernador de una provincia distante, poco conocido por el público- carecía de experiencia con los grandes medios. Por ello, el gobierno decidió obviar al periodismo como trasmisor de sus mensajes, al tiempo que desplegó un dispositivo de comunicación directa con la ciudanía. Este mecanismo , bautizado como el "atril asesino", consistía en utilizar actos oficiales, inauguraciones y recepciones presidenciales para instalar mensajes públicos en forma unidireccional y controlada. Paralelamente, se implementó 
un estricto control vertical de filtraciones informativas hacia el periodismo, provenientes del núcleo gubernamental (Kitzberger 2016).

En contraste, las decisiones referentes a la regulación del sector favorecieron a las grandes empresas de medios. A poco de asumir, el gobierno promulgó una ley que evitó la pérdida del control del Grupo Clarín S.A., entonces fuertemente endeudado, a manos de sus acreedores extranjeros. En 2005 suspendió por decreto el plazo de conteo de las licencias televisivas concedidas por diez años, luego de renovarlas por otra década a las mayores empresas. Dos años más tarde, también aprobó por decreto la fusión de las mayores operadoras de televisión por cable bajo el control del Grupo Clarín S.A.

Sin embargo, esta relación pragmática se deterioró durante el tercio final del período presidencial, lo cual se reflejó en la cobertura crítica y denunciante de los medios del grupo. Algunos observadores atribuyen este desgaste a discrepancias con la política económica, al resistido interés del multimedio por expandirse a las telecomunicaciones y a la decisión de postular a CFK como candidata presidencial en 2007 (Mochkofsky 2011; Sivak 2015).

La presidencia de CFK nació en un contexto de convivencia agriada con los grandes medios. El cambio de una estrategia de convivencia a una de confrontación, ocurrió a mediados de 2008, a raíz del conflicto agropecuario por la incorporación de retenciones móviles a las exportaciones de soja. Bajo el peso de las experiencias vividas por la clase política durante la crisis de 2001, el gobierno percibió la cobertura mediática del lock out agrario como una acción desestabilizadora. El encuadre mediático de las protestas como un "paro histórico" de "gente" movilizada de manera "espontánea" y la extensa cobertura en vivo de las movilizaciones, llevó al gobierno a pensar que los "medios dominantes" articulaban intereses por "destituirle".

El gobierno decidió colocar esta convicción en el centro de la escena. La presidenta pasó a denunciar sistemáticamente a los medios de azuzar el conflicto, desinformar, sesgar la información y buscar desestabilizar al gobierno. Incluso, en una analogía con el golpe militar de 1976, llegó a tildar a sus directivos de "generales multi-mediáticos" articuladores de mensajes "cuasi-mafiosos". Por su parte, el expresidente Kirchner se mostró con carteles que rezaban "Clarín miente" y "Todo negativo" (en alusión a la señal de noticias TN), mientras popularizó el latiguillo “¿Qué te pasa Clarín?”. El gobierno también judicializó temas que afectaban la legitimidad de las grandes empresas de noticias. Promovió la investigación por la presunta adquisición ilegítima durante la dictadura militar de Papel Prensa (empresa mixta productora de papel de diario controlada por Clarín y La Nación) y apoyó a las Abuelas de Plaza de Mayo en el esclarecimiento de la identidad de los hijos adoptivos de Ernestina Herrera de Noble, principal accionista del Grupo Clarín S.A.

Las críticas a la actuación de los grandes medios en el conflicto agrario rápidamente se multiplicaron. Personalidades de la cultura y periodistas 
visibilizaron ese accionar interesado. Varios formaron el colectivo de intelectuales Carta Abierta, que en su declaración fundacional identificó a los conglomerados mediáticos como constructores de "sentidos dominantes". Este apoyo, minoritario pero significativo, contribuyó a expandir la idea de que los grandes medios construyen interesadamente la realidad y ostentan un poder excesivo (Mochkofsky 2011).

El gobierno de CFK también radicalizó la comunicación directa y evitó las escenas mediáticas controladas por el periodismo. Para ello, incrementó el uso de la cadena nacional- de ocho veces en 2008, a 22 en 2012-, cuando Kirchner apenas la había utilizado en dos oportunidades (Vommaro y Schuliaquer 2014); multiplicó la transmisión de actos de gobierno; emplazó spots en el programa Fútbol para Todos, la estatizada transmisión deportiva que, previamente, pertenecía a un emprendimiento asociado al Grupo Clarín S.A.; y dinamizó las cuentas de Twitter y Facebook oficiales como espacio de crítica a los medios tradicionales. También proliferaron periódicos, canales de noticias y programas de radio y televisión explícitamente alineados con el gobierno, enfocados en los sesgos ideológicos y corporativos de las narrativas construidas por los medios dominantes. La mayor innovación fue la aparición de 678, programa diario en horario central de la televisión pública que utilizaba un fabuloso manejo de archivo para la deconstrucción crítica del discurso periodístico dominante. Sus informes se proponían evidenciar prejuicios clasistas y estigmatizaciones, invisibilización de temas, encuadres arbitrarios, formas de titulación y jerarquización de noticias. El impacto de 678 fue doble. Por un lado, generó repercusión en el espacio mediático que reproducía lo que el programa presentaba y, por el otro, creó un público dispuesto a movilizarse en ocasión del debate legislativo sobre la regulación mediática (Kitzberger 2016).

Como resultado, a fines de 2008 se sancionó la Ley de Servicios de Comunicación Audiovisual con un amplio apoyo de sectores de la sociedad civil. Las estrictas restricciones a la concentración de la propiedad mediática, la promoción de medios no comerciales y las orientaciones proteccionistas para las industrias culturales y los derechos de las audiencias de la norma, afectaron los intereses de los grandes actores del sector que cuestionaron judicialmente la ley.

\section{LA CONFIANZA DE LA OPINIÓN PÚBLICA EN LOS MEDIOS DE COMUNICACIÓN}

El enfrentamiento político entre el kirchnerismo y las grandes empresas de medios constituye una oportunidad única para analizar en qué medida, los encuadres y marcos interpretativos emitidos por las elites políticas, influyen en la formación de las percepciones de la opinión pública. Como explican varios trabajos recientes, en América Latina preexistían movimientos reformistas activados en torno a la democratización de los medios de comunicación (Segura y Waisbord 2016; Kitzberger s/f). Pero, la cuestión del rol de los medios en la 
dinámica democrática nunca había estado presente en la agenda pública. Su inclusión fue el resultado de un proceso definido y transmitido "desde arriba" por las elites gubernamentales asociadas a los experimentos de la nueva izquierda.

Esta sección ofrece un conjunto de hipótesis empíricamente demostrables que ponen el acento en los encuadres y otros factores políticos como determinantes de la variación individual de la confianza en los medios. La discusión se nutre de investigaciones en ciencia política y comunicación social sobre credibilidad de los medios y convicción en las instituciones políticas en general.

El tema de la credibilidad en los medios ha sido objeto de un amplio debate en Estados Unidos desde la década de 1940 (Metzger et al. 2003; Golan 2010). Algunos analistas han señalado que la noción de confianza, aplicada al estudio de medios, es compleja porque comporta múltiples facetas e interrelaciones entre emisor, receptor, mensaje y canales de transmisión (Berlo, Lemert, y Mertz 1969). Sin embargo, es posible identificar dos grandes tipos de explicaciones sobre la credibilidad en los medios: las que focalizan en la fuente del mensaje y aquellas que lo hacen en los atributos de los receptores.

Para las primeras, ciertas cualidades del emisor o fuente de la noticia — desde formas sustantivas como experiencia, profesionalismo, objetividad y precisión, hasta modos superficiales como apariencia- afectan los procesos de persuasión e influyen sobre la formación de actitudes individuales hacia los medios (Sundar y Nass 2001; Lafferty y Goldsmith 2004). Así, la credibilidad es entendida como una propiedad relativamente fija y objetiva de la fuente emisora.

Desafortunadamente, no existen datos en Argentina sobre atributos específicos de los medios y calidad de cobertura que permitan examinar si (y cómo) la opinión pública cree que una fuente es confiable o más/menos confiable que otra. Siguiendo estudios previos que enfrentan esta limitación, aquí se explora indirectamente el impacto de la fuente del mensaje sobre la confianza, utilizando el grado de "sofisticación" política de los entrevistados como indicador de "calidad" del emisor (Moehler y Singh 2011; Layton 2012). La expectativa teórica es que los individuos políticamente más sofisticados —quienes expresan preocupación por la política, poseen información y conocimiento de los asuntos políticos y consumen fuentes alternativas de noticias- están más alertas a los errores, interpretaciones sesgadas y falta de objetividad de los medios y, por lo tanto, más inclinados a penalizarlos. Se presume, entonces, una relación negativa entre interés político, entendimiento sobre la política y exposición al consumo de noticias en internet (Tsfati y Cappella 2003; Jones 2004; Tsfati 2010), y la confianza en los medios tradicionales de comunicación.

Una segunda interpretación examina el rol de factores directamente vinculados con los receptores de la noticia antes que con la fuente y/o el contenido del mensaje. El punto de partida es que los receptores no son sujetos pasivos, aislados e imparciales, sino destinatarios "sociales" que evalúan al emisor e 
interpretan los mensajes según sus propios valores, actitudes y experiencias (Gunther 1992; Metzger et al. 2003). A diferencia de la interpretación anterior, la credibilidad es entendida como el resultado de un juicio subjetivo y contextual de quienes reciben el mensaje (Tsfati 2003).

Dentro de la literatura que analiza el papel de la subjetividad de las actitudes individuales hacia los medios, una de las corrientes más dinámicas está representada por los estudios del llamado "efecto hostil de los medios". Este fenómeno refiere al hecho de que los portadores de creencias políticas intensas (o partidarios) sobre un determinado tema, tienden a percibir la cobertura mediática de cuestiones sensibles y controversiales vinculadas a esa idea como injusta y hostilmente sesgada contra sus puntos de vista (Vallone, Ross, y Lepper 1985; Mason y Nass 1989; D’Alessio 2003).

El fenómeno de la hostilidad mediática ha sido testeado en una variedad de tópicos, incluyendo las elecciones presidenciales (Dalton, Beck, y Huckfeldt 1998), el suicidio asistido (Gunther y Christen 2002), los alimentos transgénicos (Schmitt, Gunther, y Liebhart 2004) y las noticias deportivas (Arpan y Raney 2003). Más vinculado con las preocupaciones de este trabajo, una legión de estudios en Estados Unidos ha explorado la relación entre hostilidad mediática, ideología política y partidismo. Esta literatura reporta que las personas con ideologías conservadoras así como los republicanos, perciben la presencia de un sesgo hostil - liberal y/o prodemócrata- en los medios y, por lo tanto, manifiestan menores niveles promedio de confianza (Jones, 2004; Cook y Gronke 2007; Lee 2010). Esta asociación se explica porque, en Estados Unidos, el periodismo es considerado una profesión "liberal" y se supone, entonces, que los medios sesgan la cobertura de noticias hacia la izquierda.

Sin embargo, en Argentina y el resto de América Latina, el vínculo entre hostilidad mediática e ideología política debería operar en sentido diferente. La propiedad de los conglomerados de medios, en la región, ha estado históricamente ligada a poderosas élites políticas y económicas. La concentración de la propiedad en sectores del establishment y grandes corporaciones privadas, explica que los partidos populistas clásicos y las diferentes expresiones de izquierda hayan sido portadores de discursos críticos y agendas reformistas sobre los medios. Ciertamente, las orientaciones conservadoras también han sido un atributo de los propietarios de medios en Estados Unidos. Pero durante el siglo xx, las redacciones periodísticas norteamericanas fueron conquistando una gran autonomía profesional (Hallin y Mancini 2004). En contraste, el periodismo latinoamericano - aun el que representa orientaciones progresistas - ostenta un bajo grado de profesionalización e independencia respecto de los propietarios de medios. Éstos conservan una fuerte injerencia en la determinación de las decisiones editoriales, lo cual ha perpetrado la tendencia a concebir y utilizar los medios como instrumentos de los intereses políticos o corporativos de sus dueños (Waisbord 2000). En vista de estas características, en Argentina se espera encontrar mayores niveles de confianza entre quienes se identifican con ideologías de derecha. 
Complementando el razonamiento de la hostilidad mediática con la literatura sobre partidismo político, varios estudios sugieren que los partidarios del gobierno expresan menor confianza en los medios debido a que éstos actúan como sujetos de observancia y contraloría pública (Cook, Gronke, y Rattliff 2000; Cook y Gronke 2007). Naturalmente, en este trabajo se espera que, quienes manifiestan apoyo al gobierno kirchnerista, confíen sistemáticamente menos en los medios de comunicación.

La hipótesis central de este trabajo, sin embargo, es que el efecto de la afinidad política hacia el gobierno opera diferente entre votantes y simpatizantes. Esto es así porque los votantes desarrollan un vínculo evaluativo -y, por lo tanto, cambiante- con los gobiernos en base a percepciones y consideraciones coyunturales. En cambio, los simpatizantes construyen un vínculo normativo e identitario permanente con los gobiernos, y es esperable que también compartan sus cosmovisiones políticas. Los simpatizantes del kirchnerismo deberían, entonces, compartir la interpretación del gobierno acerca de la naturaleza política (no-neutral) y corporativamente sesgada de los conglomerados mediáticos, por lo que se espera que expresen niveles significativamente menores de confianza. Por otra parte, la credibilidad expresada por los votantes será condicional a la evaluación que tengan del desempeño presidencial: cuanto menor/mayor sea esta consideración, mayor/menor será la confianza en las empresas mediáticas.

Finalmente, para completar la discusión, es relevante indagar el potencial efecto de temas controversiales para el gobierno, sensibles para la opinión pública y de extensa cobertura mediática. Dos candidatos naturales son la corrupción y la criminalidad. Los datos indican que, alrededor del 37\% de los argentinos, declaran que alguien en su hogar fue víctima de un delito en el último año, mientras que cerca del $22 \%$ manifiesta haber sido victimizado en un acto de corrupción. En este sentido, un grupo de trabajos muestran que las víctimas de la corrupción y la delincuencia confían menos en las instituciones y actores del sistema democrático (Seligson 2006; Weitz-Shapiro 2008; Booth y Seligson 2009; Bateson 2010; Carreras 2013). Debido a que las personas victimizadas suelen culpar al gobierno por la falta de transparencia y seguridad, y los medios tienden a reportar regularmente estos fenómenos de manera escandalosa (Martini 2007), se espera que las víctimas del crimen y la corrupción expresen mayor confianza en las instituciones mediáticas que las no-víctimas.

\section{DATOS Y MÉTODOS}

¿Cuáles son los determinantes políticos y sociológicos de la variación individual en los niveles de confianza de los argentinos en los medios de comunicación? ¿Hasta qué punto los recursos mediáticos y encuadres discursivos desplegados por el gobierno kirchnerista afectaron la credibilidad de los ciudadanos en los grandes medios? Para responder estas interrogantes se utilizan los datos de opinión pública generados por las rondas 2008, 2010, 2012 y 2014 del Barómetro 
de las Américas. Las cerca de 6.000 personas entrevistadas en Argentina recibieron la siguiente pregunta, que aquí sirve como variable dependiente: “¿Hasta qué punto tiene usted confianza en los medios de comunicación?” Las respuestas, originariamente provistas en una escala de 1 a 7 , donde 1 significa 'nada' y 7 significa 'mucho', fueron recodificadas para facilitar la interpretación a una escala de 0 a 100 puntos con mayores valores indicando mayor confianza. ${ }^{2}$

Es importante mencionar que la medida de confianza en los medios utilizada en este trabajo presenta una serie de limitaciones. El primer problema refiere a la noción general de "medios de comunicación" empleada en la pregunta, para evitar sesgos de deseabilidad e inducción de respuestas. Es de esperar que la neutralidad de la consigna induzca en los encuestados una noción de medios que se restringe a las grandes organizaciones mediáticas con mayor presencia en el mercado de noticias. Segundo, la pregunta no captura diferentes formas de confianza, incluyendo la confianza en la selección de temas y hechos, en la precisión de las descripciones y en las evaluaciones periodísticas (Metzger et al. 2003; Kohring y Matthes 2007). Este trabajo adopta un enfoque pragmático sobre el tema, que evita el debate sustancial en torno a la naturaleza de la confianza. Se asume que la confianza en los medios es una forma especial de certeza y que los respondientes forman sus opiniones acerca de cuán creíble es un medio comparando lo que saben sobre él con sus expectativas respecto de cómo debería ser. Por último, también es problemático la falta de un indicador que permita distinguir niveles de confianza entre diferentes proveedores de noticias y entre diferentes tipos de medios. Existe, por ejemplo, evidencia de que los ciudadanos de países africanos confían más en la información generada por medios de propiedad pública que privada, aun luego de controlar por niveles de libertad de expresión y corrupción (Moehler y Singh 2011). Por otro lado, varios trabajos en Estados Unidos muestran que los individuos expresan diferentes niveles de confianza en diferentes tipos de medios como la televisión (Bucy 2003), los periódicos (Cassidy 2005) y los medios en línea. Así, medidas más refinadas de confianza permitirían investigar estas y otras cuestiones relevantes en Argentina. Pero, infelizmente, estas medidas no existen.

Vale la pena, también, señalar que el tipo de datos —encuestas de corte transversal- utilizados en este estudio, tiene limitaciones en comparación con otras alternativas. Hubiera sido deseable contar con datos individuales de panel para identificar si los cambios operados en la confianza de los ciudadanos en los medios fueron inducidos por señales emitidas por las élites de gobierno o por otros factores. Alternativamente, podría haberse administrado un experimento de lista en la encuesta para observar en qué medida y cómo las élites modelan la opinión pública a través del uso de marcos interpretativos y retóricos. De todas maneras, contamos con datos a nivel individual recolectados antes y después de que estallara el epicentro del conflicto entre el gobierno y los grandes medios. Esta condición permite realizar argumentos causales acerca del efecto 
de los encuadres y narrativas elaboradas por las élites gubernamentales que son independientes de la simpatía partidaria declarada por los encuestados.

Para examinar empíricamente los argumentos discutidos en la sección anterior, se estimaron modelos oLs (del inglés Ordinary Least Squares) con parámetros corregidos por el diseño complejo de la muestra. Debido a que la variable dependiente es categórica y construida a partir de una escala de Likert, algunos metodólogos sostienen que ols es un método inapropiado y recomiendan utilizar modelos logísticos ordenados. Sin embargo, una serie de estudios demuestran que, bajo ciertas condiciones (sesgo moderado y número suficiente, al menos 7, de intervalos), las escalas de Likert pueden analizarse de manera robusta con modelos lineales (Carifio y Perla 2007; Norman 2010). Aun cuando nuestra variable dependiente cumple con las condiciones de linealidad, también se estimaron modelos logísticos ordenados con idénticos resultados. ${ }^{3}$

El primer conjunto de variables independientes sirve para testear el argumento según el cual, los individuos políticamente sofisticados expresan menores niveles promedio de confianza en los medios. Existen varias combinaciones de variables que capturan la idea de "sofisticación" política. En este trabajo, se consideran los siguientes tres componentes: interés en la política, "eficacia política interna" o el grado en el cual un individuo cree entender los temas importantes de su país (Craig, Niemi, y Silver 1990), y el uso de internet para informarse. ${ }^{4}$ La Tabla 1 presenta la estadística descriptiva de estas variables, así como de las que se discuten a continuación.

Estos modelos no se reportan por cuestiones de espacio y están disponibles a pedido de los lectores. Las preguntas utilizadas para construir estas variables son las siguientes. Interés en la política: “¿Qué tanto interés tiene usted en la política?" Mucho, algo, poco, nada. Eficacia interna: “¿Usted siente que entiende bien los asuntos políticos más importantes del país?" Escala 1 a 7 con valores más altos indicando mayor acuerdo. Uso de internet: “QQué tan frecuentemente usa internet para informarse?” Diariamente, algunas veces a la semana, algunas veces al mes, rara vez, nunca. Todas estas variables fueron recodificadas a una escala de 0 a 100 puntos para facilitar su interpretación. 
Tabla 1. Estadística descriptiva de las variables incluidas en los modelos

\begin{tabular}{|c|c|c|c|c|}
\hline Variable & Media & $\begin{array}{l}\text { Desviación } \\
\text { estándar }\end{array}$ & Mínimo & Máximo \\
\hline Confianza en los medios & $\begin{array}{l}54,55 \\
55,86\end{array}$ & $\begin{array}{l}28,28 \\
29,81\end{array}$ & 0 & 100 \\
\hline Simpatizante Frente para la Victoria & $\begin{array}{l}, 056 \\
, 112\end{array}$ & $\begin{array}{l}\text {,230 } \\
\text {,315 }\end{array}$ & 0 & 1 \\
\hline Votante CFK & $\begin{array}{l}, 362 \\
, 584\end{array}$ & $\begin{array}{l}481 \\
, 493\end{array}$ & 0 & 1 \\
\hline Aprobación presidencial & $\begin{array}{l}54,11 \\
50,61\end{array}$ & $\begin{array}{l}20,47 \\
27,18\end{array}$ & 0 & 100 \\
\hline Ideología & $\begin{array}{l}5,52 \\
5,48\end{array}$ & $\begin{array}{l}1,86 \\
2,00\end{array}$ & 1 & 10 \\
\hline Eficacia interna & $\begin{array}{l}47,44 \\
47,87\end{array}$ & $\begin{array}{l}29,60 \\
31,12\end{array}$ & 0 & 100 \\
\hline Interés en la política & $\begin{array}{l}38,84 \\
61,16\end{array}$ & $\begin{array}{l}33,02 \\
33,02\end{array}$ & 0 & 100 \\
\hline Victimización por corrupción & $\begin{array}{l}27,46 \\
19,73\end{array}$ & $\begin{array}{l}44,64 \\
39,80\end{array}$ & 0 & 1 \\
\hline Victimización por crimen & $\begin{array}{l}39,45 \\
39,89\end{array}$ & $\begin{array}{l}48,88 \\
48,97\end{array}$ & 0 & 1 \\
\hline Confianza institucional & $\begin{array}{l}235,30 \\
263,75\end{array}$ & $\begin{array}{l}118,90 \\
131,32\end{array}$ & 0 & 600 \\
\hline Edad & $\begin{array}{l}39,9 \\
38,8\end{array}$ & $\begin{array}{l}16,70 \\
16,04\end{array}$ & $\begin{array}{l}16 \\
18\end{array}$ & $\begin{array}{l}89 \\
81\end{array}$ \\
\hline Educación & $\begin{array}{l}10,38 \\
10,44\end{array}$ & $\begin{array}{l}3,95 \\
3,97\end{array}$ & $\begin{array}{l}0 \\
0\end{array}$ & $\begin{array}{l}22 \\
18\end{array}$ \\
\hline Riqueza & $\begin{array}{l}2,95 \\
2,95\end{array}$ & $\begin{array}{l}1,42 \\
1,42\end{array}$ & 1 & 5 \\
\hline Tamaño del lugar de residencia & $\begin{array}{l}2,75 \\
1,85\end{array}$ & $\begin{array}{l}1,09 \\
1,07\end{array}$ & 0 & 4 \\
\hline
\end{tabular}

Nota. El primer valor en las casillas corresponde a la ronda 2008 y el segundo a las de 2010, 2012 y 2014.

El segundo conjunto de variables permite evaluar las hipótesis de mayor interés. Por un lado, todos los modelos, alternativamente, incluyen variables dicotómicas que identifican a quienes votaron por CFK en las elecciones presidenciales de 2007 (para los encuestados en 2008 y 2010) y 2011 (para los encuestados en 2012 y 2014), y a quienes declaran simpatizar con el partido de gobierno (Frente Para la Victoria, FPV). ${ }^{5}$ Obviamente, se espera encontrar una relación negativa entre estas variables y el nivel de confianza en los medios. Este efecto debería ser mayor entre los simpatizantes del FPV, quienes comparten un vínculo identitario y estable con el gobierno, que entre los votantes de CFK, quienes cultivan una

5 Las encuestas se administraron luego de las elecciones presidenciales. Esto puede generar distorsiones, ya que las encuestas postelectorales suelen reportar niveles de apoyo al ganador superiores al porcentaje de 
relación evaluativa y circunstancial. De hecho, como se anticipó, se espera que la confianza de los votantes sea condicional a la evaluación del desempeño presidencial. Para testear esta hipótesis, se incluyen modelos con un término de interacción entre ambas variables.

Por otro lado, todas las estimaciones incorporan una variable de Ideología política para evaluar el argumento que vincula ideologías conservadoras con mayores niveles de confianza en los medios. Como es norma en los estudios sobre comportamiento político, el indicador de ideología es el auto-posicionamiento de los respondientes en una escala de 1 a 10, donde 1 significa 'izquierda' y 10 'derecha'.6

Respecto de las variables de Corrupción y Criminalidad utilizadas para medir el impacto de temas controversiales para el gobierno, se adoptaron las preguntas de la encuesta que refieren a experiencias individuales de victimización por estos delitos. ${ }^{7}$ La victimización por corrupción se mide con una batería de preguntas que indagan en el encuestado si debió pagar un soborno en diferentes instancias durante el último año. La victimización por crimen resulta de preguntar si algún miembro del hogar fue víctima de un acto delincuencial (robo, hurto, agresión, fraude, chantaje, extorsión o amenazas) en ese periodo.

Debido a que la confianza en los medios puede ser parte de una confianza más generalizada en otras instituciones (Newton y Norris 2000), los modelos incluyen una variable de Confianza Institucional generalizada, basada en el nivel de confianza declarada en seis instituciones políticas: las Fuerzas Armadas, los partidos políticos, la Iglesia católica, el Congreso, los intendentes y la policía. ${ }^{8}$ Se entiende que esta variable refleja una tendencia general de confianza individual en las instituciones, relacionada pero separada de una tendencia particular de confianza en los medios. De hecho, la correlación entre ambas variables es considerablemente baja $(r=, 415)$.

Por último, todos los modelos incluyen una serie de variables socio-demográficas que están presumiblemente relacionadas con variaciones en el nivel de confianza de la opinión pública en los medios de comunicación: edad, género, educación (número de años de enseñanza formal), riqueza (índice de bienes en el hogar compuesto por cinco quintiles) y tamaño del lugar de residencia. Estudios previos reportan que la edad, la riqueza y la educación están negativamente

votos obtenido. En este trabajo, el sesgo es leve: el voto a CFK está sobrestimado en solo $10 \%$. Es importante, además, notar que varios entrevistados no declaran su voto, reduciendo el número de observaciones. Sin embargo, no hay razones para suponer que quienes votaron al oficialismo están más o menos inclinados a reportar su voto. La tasa de no respuesta/no sabe es de $35,5 \%$.

Debido a que los valores 5 y 6 de la escala pueden reflejar situaciones de no-respuesta encubierta, los modelos fueron también estimados con la escala sin estos valores. Los resultados son equivalentes a los generados con la escala completa.

También se estimaron modelos con variables que miden la percepción de corrupción (o cuán generalizada cree que está la corrupción) y la percepción de inseguridad (o cuán inseguro se siente en su barrio). En ninguna instancia, estas variables alcanzaron significancia estadística.

$8 \quad$ El alpha de Cronbach de esta variable es altamente confiable: $\alpha .772$. 
relacionados con la confianza en las instituciones mediáticas (Cappella y Jamieson 1997), mientras que las mujeres confían más (Layton 2012).

\section{RESULTADOS}

Los resultados de los modelos ols presentados en la Tabla 2 y la Tabla 3 confirman, en gran medida, las expectativas teóricas. La Tabla 2 muestra las estimaciones para la ronda 2008 que se realizó cinco meses antes de que estallara el enfrentamiento entre el gobierno y los grandes medios, a propósito de la cobertura del lock out agrario. La Tabla 3 presenta los resultados de los mismos modelos para las rondas 2010, 2012 y 2014 que fueron realizadas bastante tiempo después, luego de que los dispositivos comunicacionales y encuadres retóricos del gobierno estaban instalados en la opinión pública. En ambas tablas, el Modelo 1 corresponde a las estimaciones calculadas para los simpatizantes del FPV. El Modelo 2 para los votantes de CFK. El Modelo 3 incorpora el término de interacción entre votantes de CFK y aprobación presidencial para testear la hipótesis condicional.

Tabla 2. Regresión oLs. Previo al conflicto con los medios (ronda 2008)

\begin{tabular}{|c|c|c|c|}
\hline Variables & Modelo 1 & Modelo 2 & Modelo 3 \\
\hline \multicolumn{4}{|l|}{ Preferencias políticas } \\
\hline Votante CFK & & $\begin{array}{r}, 282 \\
(2,57)\end{array}$ & $\begin{array}{l}-2,80 \\
(9,02)\end{array}$ \\
\hline Simpatizante FPV & $\begin{array}{l}-6,54 \\
(4,22)\end{array}$ & & \\
\hline Aprobación presidencial & $\begin{array}{r}, 060 \\
(, 048)\end{array}$ & $\begin{array}{r}, 030 \\
(, 061)\end{array}$ & $\begin{aligned}, 014 \\
(, 071)\end{aligned}$ \\
\hline $\begin{array}{l}\text { Votante CFK * } \\
\text { Aprobación presidencial }\end{array}$ & & & $\begin{array}{r}, 051 \\
(, 140)\end{array}$ \\
\hline Ideología & $\begin{array}{l}1,13^{*} \\
(, 507)\end{array}$ & $\begin{array}{r}, 786 \\
(, 612)\end{array}$ & $\begin{array}{r}, 780 \\
(, 614)\end{array}$ \\
\hline \multicolumn{4}{|l|}{ Sofisticación política } \\
\hline Eficacia interna & $\begin{array}{r}, 036 \\
(, 036)\end{array}$ & $\begin{array}{r}, 013 \\
(, 041)\end{array}$ & $\begin{array}{r}, 012 \\
(, 041)\end{array}$ \\
\hline Interés político & $\begin{array}{r}-, 089^{* *} \\
(, 034)\end{array}$ & $\begin{array}{r}-, 106^{* *} \\
(, 038)\end{array}$ & $\begin{array}{r}-, 106^{* *} \\
(, 038)\end{array}$ \\
\hline Uso de internet & $\begin{array}{l}-, 056^{*} \\
(, 028)\end{array}$ & $\begin{array}{l}-, 078^{*} \\
(, 034)\end{array}$ & $\begin{array}{l}-, 078^{*} \\
(, 034)\end{array}$ \\
\hline Educación & $\begin{array}{r}-, 356 \\
(, 282)\end{array}$ & $\begin{array}{r}-, 049 \\
(, 331)\end{array}$ & $\begin{array}{r}-, 043 \\
(, 331)\end{array}$ \\
\hline
\end{tabular}




\begin{tabular}{lrrr}
\hline Temas & & & \\
\hline Victimización por corrupción &,- 013 &,- 009 &,- 009 \\
& $(, 019)$ & $(, 023)$ & $(, 023)$ \\
Victimización por crimen &, 029 &, 017 &, 018 \\
& $(, 019)$ & $(, 022)$ & $(, 022)$ \\
Confianza institucional &, $107^{* * *}$ &, $109^{* * *}$ &, $109^{* * *}$ \\
& $(, 009)$ & $(, 010)$ & $(, 010)$ \\
Edad &,- 000 &,- 006 &,- 0443 \\
& $(, 071)$ & $(, 331)$ & $(, 331)$ \\
Mujer & $3,84^{*}$ & 1,16 & 1,14 \\
& $(1,82)$ & $(2,09)$ & $(2,09)$ \\
Riqueza &, 443 &, 446 &, 434 \\
Lugar de residencia & $(, 890)$ & $(, 944)$ & $(, 947)$ \\
\hline Constante &, 968 &, 295 &, 297 \\
R2 & $(, 589)$ & $(1,06)$ & $(1,06)$ \\
F test & $20,15^{* *}$ & $26,38^{* * *}$ & $27,14^{* *}$ \\
Prob> F & $(7,24)$ & $(8,11)$ & $(8,69)$ \\
N &, 271 &, 270 &, 270 \\
\hline
\end{tabular}

Notas. Variable dependiente es índice de confianza en los medios. Errores estándar robustos entre paréntesis. ${ }^{* * *}$ $\mathrm{p}<, 001 ;{ }^{* *} \mathrm{p}<, 01 ;{ }^{*} \mathrm{p}<, 05$.

Tabla 3. Regresión oLs. Posterior al conflicto con los medios (rondas 2010, 2012, 2014)

\begin{tabular}{lrrr}
\hline \multicolumn{1}{c}{ Variables } & Modelo 1 & Modelo 2 & \multicolumn{1}{c}{ Modelo 3 } \\
\hline Preferencias políticas & & & \\
\hline Votante CFK & & 1,91 & $12,10^{* * *}$ \\
& $-5,51^{* *}$ & $(1,58)$ & $(3,65)$ \\
Simpatizante FPV & $(2,21)$ & & \\
&,- 025 &,$- 097^{* *}$ &,- 004 \\
Aprobación presidencial & $(, 027)$ & $(, 032)$ & $(, 046)$ \\
& & &,$- 186^{* *}$ \\
Votante CFK * & & & $(, 061)$ \\
Aprobación presidencial & $1,26^{* * *}$ &, $771^{* *}$ &, $826^{* *}$ \\
Ideología & $(, 331)$ & $(, 373)$ & $(, 374)$ \\
\hline Sofisticación política & & &,- 029 \\
\hline Eficacia interna &,- 031 &,- 036 & $(, 025)$
\end{tabular}




\begin{tabular}{|c|c|c|c|}
\hline Interés político & $\begin{array}{r}-, 100^{* * *} \\
(, 021)\end{array}$ & $\begin{array}{r}-, 129^{* * *} \\
\quad(, 024)\end{array}$ & $\begin{array}{r}-, 127^{* * *} \\
(, 024)\end{array}$ \\
\hline Uso de internet & $\begin{array}{r}-, 055^{* *} \\
(, 019)\end{array}$ & $\begin{array}{r}-, 056^{* *} \\
(, 021)\end{array}$ & $\begin{array}{r}-, 058^{* * *} \\
(, 021)\end{array}$ \\
\hline Educación & $\begin{array}{r}-, 639^{* * *} \\
(, 185)\end{array}$ & $\begin{array}{r}-, 729^{* * *} \\
(, 200)\end{array}$ & $\begin{array}{r}-, 730^{* * * *} \\
(, 199)\end{array}$ \\
\hline \multicolumn{4}{|l|}{ Temas } \\
\hline Victimización por corrupción & $\begin{array}{r}-, 021 \\
(, 015)\end{array}$ & $\begin{array}{r}-, 015 \\
(, 017)\end{array}$ & $\begin{array}{r}-, 013 \\
(, 017)\end{array}$ \\
\hline Victimización por crimen & $\begin{array}{l}, 035^{* *} \\
(, 012)\end{array}$ & $\begin{array}{l}, 039^{* *} \\
(, 014)\end{array}$ & $\begin{array}{l}, 037^{* *} \\
(, 014)\end{array}$ \\
\hline Confianza institucional & $\begin{array}{r}, 086^{* * *} \\
(, 005)\end{array}$ & $\begin{array}{r}, 083^{* * *} \\
(, 006)\end{array}$ & $\begin{array}{r}, 083^{* * *} \\
(, 006)\end{array}$ \\
\hline Edad & $\begin{array}{l}-, 055 \\
(, 044)\end{array}$ & $\begin{array}{l}-, 043 \\
(, 050)\end{array}$ & $\begin{array}{l}-, 038 \\
(, 050)\end{array}$ \\
\hline Mujer & $\begin{array}{r}2,01 \\
(1,21)\end{array}$ & $\begin{array}{r}2,25 \\
(1,37)\end{array}$ & $\begin{array}{r}2,26 \\
(1,36)\end{array}$ \\
\hline Riqueza & $\begin{array}{l}, 675 \\
(, 487)\end{array}$ & $\begin{array}{l}, 208 \\
(, 569)\end{array}$ & $\begin{array}{l}\text {, 331 } \\
(, 571)\end{array}$ \\
\hline Lugar de residencia & $\begin{array}{r}, 537 \\
(, 598)\end{array}$ & $\begin{array}{l}, 765 \\
\text { (,682) }\end{array}$ & $\begin{array}{l}, 717 \\
\text { (,681) }\end{array}$ \\
\hline Constante & $\begin{array}{r}41,31^{* * *} \\
(4,33)\end{array}$ & $\begin{array}{r}50,93^{* * *} \\
(5,12)\end{array}$ & $\begin{array}{r}44,27^{* * *} \\
(5,78)\end{array}$ \\
\hline R2 & ,209 & ,217 & ,222 \\
\hline F test & $\begin{array}{r}37,74 \\
0,0000\end{array}$ & $\begin{array}{r}28,43 \\
0,0000\end{array}$ & $\begin{array}{r}26,87 \\
0,0000\end{array}$ \\
\hline $\begin{array}{l}\text { Prob }>\text { F } \\
\text { N }\end{array}$ & $\begin{array}{r}0,0000 \\
1910\end{array}$ & $\begin{array}{r}0,0000 \\
1387\end{array}$ & 1387 \\
\hline
\end{tabular}

Notas. Variable dependiente es índice de confianza en los medios. Errores estándar robustos entre paréntesis. *** $\mathrm{p}<, 001 ;{ }^{* *} \mathrm{p}<, 01 ;{ }^{*} \mathrm{p}<, 05$.

Comenzando por las variables de identificación política, la evidencia ratifica las expectativas teóricas. En ninguno de los modelos estimados para la ronda 2008, es decir antes de que se visibilizara el conflicto con los conglomerados mediáticos, existe una asociación entre apoyar políticamente al kirchnerismo y expresar menores niveles de confianza en los medios. Tanto la variable que identifica a los Simpatizantes del FPV como la que identifica a los Votantes de CFK (de forma aislada e interactuada), no obtiene el nivel mínimo de significancia estadística. Tampoco es significativo el efecto de la aprobación presidencial, mientras que el impacto de la ideología solo es significativo en la dirección predicha en el Modelo 1.

Por el contrario, las estimaciones de los modelos presentados en la Tabla 3, que corresponden a los años posteriores al inicio del conflicto con los medios, una vez que los encuadres interpretativos y dispositivos comunicacionales generados desde el gobierno estaban instalados en la opinión pública, confirman las 
hipótesis centrales de este trabajo. Primero, el análisis sustancia empíricamente el argumento que asocia la identificación política con el partido de gobierno con menores niveles de confianza relativa. Como se observa en la Tabla 3, la variable Simpatizante FPV se comporta en la dirección negativa esperada y constituye el predictor más importante de la confianza en los medios. Este resultado sugiere que una parte considerable de la población pasó a evaluar el comportamiento de los conglomerados mediáticos a través de un prisma que recuperaba la construcción expresada por el kirchnerismo. ${ }^{9}$

También encuentra sustento empírico la hipótesis de condicionalidad que vincula la confianza en los medios expresada por los votantes de CFK, con la evaluación de la labor presidencial. Nótese que en el Modelo 2 (Tabla 3), donde solo se testea el efecto directo de los votantes, esta variable tiene signo positivo (lo cual sugiere que confían más que los opositores), aunque no es estadísticamente significativa. Para evaluar si existe un efecto condicional de los votantes, el Modelo 3 incorpora un término de interacción. La variable Votantes CFK continúa siendo positiva y es estadísticamente significativa. Esto significa que, cuando el nivel de aprobación presidencial es cero (o desaprobación), quienes votaron a CFK expresan mayor confianza en los medios. Dicho de otro modo, este caso representa al votante que "abandonó" al gobierno porque entiende, entre otras cosas, que su gestión es pobre. La variable de interacción, no obstante, tiene signo negativo y es significativa a nivel ,001. Esto significa que el aumento de la aprobación presidencial está asociado con una caída en la confianza en los medios. Concretamente, el coeficiente indica que, por cada punto de aumento en la aprobación del desempeño de CFK, la pendiente del efecto marginal del votante sobre la confianza en los medios disminuye en ,186.

Esta relación se observa claramente en la Figura 1, donde se ilustra por medio de una línea llena el efecto marginal (junto con los intervalos de confianza al 95\% en líneas punteadas) del voto a CFK (eje Y) en el rango de valores de aprobación presidencial (eje X). Primero, se aprecia que, a niveles relativos de aprobación (igual o menor a 48 puntos), el efecto marginal del votante kirchnerista es positivo y significativo, lo cual indica mayor confianza en los medios. Segundo, a medida que aumenta el nivel de aprobación presidencial, el efecto marginal disminuye hasta transformarse en negativo, pero sin llegar a ser estadísticamente distinguible de cero.

El efecto del partidismo es diferente del efecto de la confianza institucional, ya que ambas variables son estadísticamente significativas cuando se las incorpora de manera simultánea en los modelos y cuando se las incluye aisladamente. 
Figura 1. Efecto condicional votante cfk según aprobación presidencial

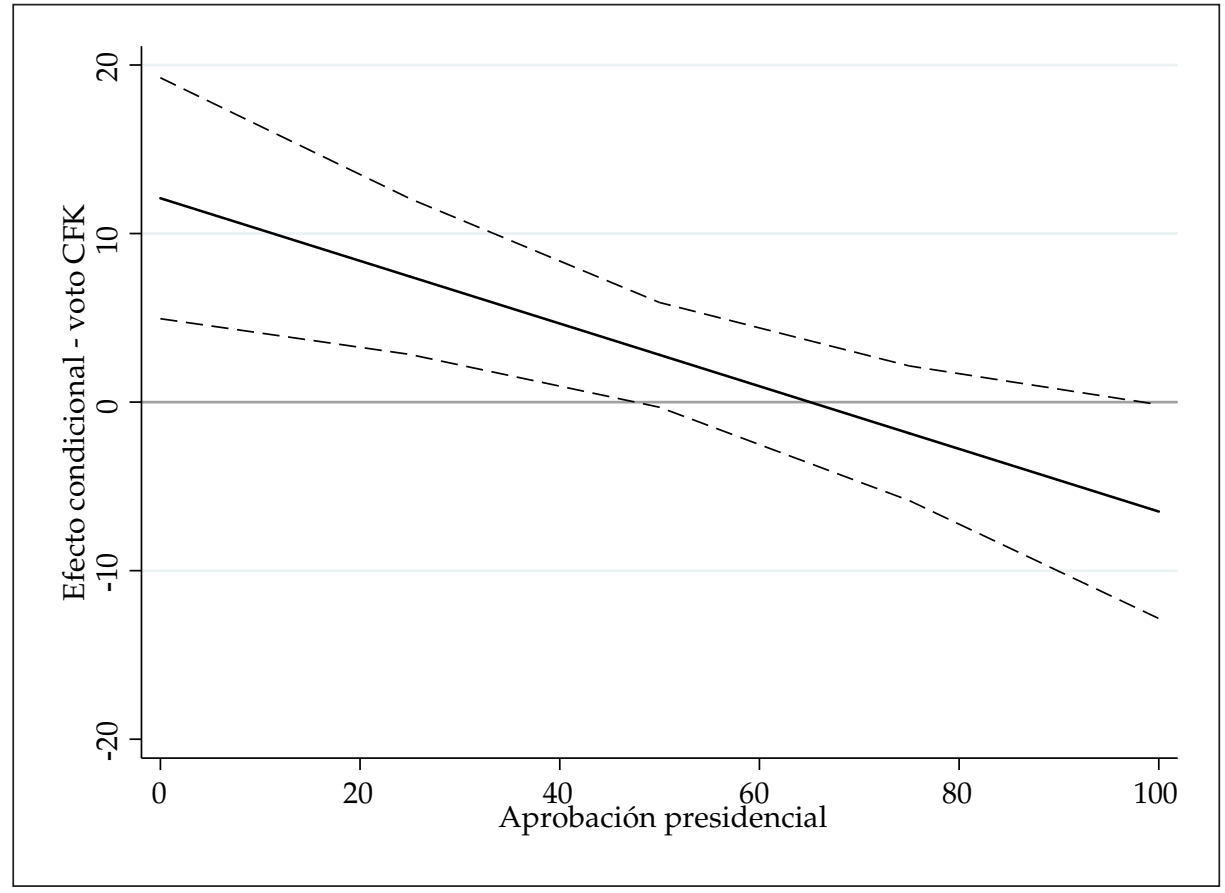

Para completar el análisis de las preferencias políticas, es importante notar que la variable Ideología no tiene significancia estadística en los modelos que capturan el clima de la opinión pública con anterioridad al conflicto con los medios, pero tiene el signo positivo esperado y es estadísticamente significativa a nivel ,001 en los modelos posteriores. Es decir, a diferencia de lo que ocurre en Estados Unidos, los argentinos que tienen ideologías políticas conservadoras expresan un mayor nivel de confianza en los medios de comunicación. Así, una persona que se identifica como 'de derecha' obtiene, en promedio, entre 8 y 13 puntos más en la escala de confianza que aquella que se identifica como 'de izquierda'.

Por otra parte, los datos abonan la hipótesis de la Sofisticación política. Es decir, las personas más sensibles a la calidad de la información confían sistemáticamente menos en los medios, aun controlando por el nivel general de confianza institucional que, naturalmente, es un predictor positivo y significativo. Así, los individuos que declaran mayor interés en los asuntos públicos, quienes consumen canales alternativos de información como internet y los más educados, expresan menor nivel de credibilidad. Esto puede deberse al hecho de que tales personas son capaces de descubrir la pobre calidad, el sensacionalismo y el sesgo informativo de los medios, penalizando su accionar como guardianes de la vida pública. Sustantivamente, las estimaciones de la Tabla 3 indican que por cada diez puntos de aumento en el índice de interés en la política (escala de 0 a 100 puntos) y por cada categoría de aumento en la frecuencia de uso de 
internet, la confianza en los medios decrece 1,3 y 0,6 puntos, respectivamente. En el mismo sentido opera el efecto de la educación, aunque llamativamente esta variable no es significativa en las estimaciones de la ronda previa a que estallara el conflicto con los medios. Como se observa en la Tabla 3, por cada año adicional de educación formal que tiene una persona, su nivel de confianza decrece entre 0,64 y 0,73 puntos, dependiendo del modelo. Es interesante notar, por su parte, que la eficacia política interna no tiene efecto sobre la credibilidad. Esto puede deberse a un sesgo de deseabilidad social, en el que los encuestados responden que entienden los problemas políticos importantes en lugar de confesar que no los comprenden.

Por último, la evidencia muestra que, previo al desencadenamiento del conflicto con los medios, las variables que capturan la victimización por crimen y corrupción no tienen impacto sobre los niveles de confianza. Pero en el periodo posterior, las víctimas del crimen —aunque no así de la corrupción-manifiestan confiar más en los medios que las no-víctimas. De todas maneras, el tamaño de los coeficientes es relativamente bajo, lo cual sugiere que el tratamiento de estos temas sensibles, por parte de los medios, no parece afectar seriamente su credibilidad ante la opinión pública. Nótese, además, que ninguna variable sociodemográfica tiene significancia estadística.

Como sucede en la mayoría de las investigaciones que examinan los determinantes individuales de la credibilidad en las instituciones políticas, los modelos de regresión explican solamente una pequeña porción de la varianza en la confianza en los medios: el R2 varía entre ,209 y ,222. Esto significa que otros factores relevantes permanecen ocultos y deben ser explorados. En especial, aquellos que remiten a la confianza en aspectos específicos de diferentes tipos de medios y a los mecanismos de persuasión elaborados por las élites gubernamentales. Asimismo, las estimaciones demuestran la existencia de una asociación entre variables políticas y la confianza en los medios, pero no siempre es clara la dirección de la causalidad. En algunos casos, esta dirección es obvia: es improbable, por ejemplo, que la confianza en los medios haga que los encuestados sean más educados. Pero con otras variables, como ideología y simpatía política, el vínculo causal es más complejo y puede operar en ambas direcciones. El problema de determinar la dirección causal entre actitudes individuales y consumo de medios es frecuentemente abordado por la literatura y no puede ser resuelto con los datos disponibles para este estudio.

\section{CONCLUSIÓN}

Con la llegada al poder de la nueva izquierda, en América Latina tuvo lugar un proceso de politización del orden mediático. A pesar del interés que despertó este fenómeno, no existen trabajos que indaguen acerca del efecto de los encuadres interpretativos y la retórica de las élites gubernamentales sobre la confianza de la opinión pública en las instituciones mediáticas. El periodo de las 
administraciones kirchneristas en Argentina constituye un escenario ideal para atender este interrogante, dado que la confrontación entre el Gobierno y los medios tradicionales alcanzó un alto nivel de radicalidad. En efecto, se destacan la construcción de cuestionamientos críticos a los conglomerados mediáticos en el discurso presidencial y en dispositivos comunicacionales movilizados desde el Gobierno, y la aprobación de una ambiciosa legislación orientada a regular el funcionamiento del sector.

La evidencia empírica confirma que los encuadres y narrativas del gobierno kirchnerista acerca de la naturaleza políticamente interesada y sesgada de las grandes empresas de medios, afectaron a una porción significativa de la población. Así, los simpatizantes del kirchnerismo confían menos en los medios que el resto de las personas, mientras que los votantes de CFK observan la labor presidencial a la hora de evaluar la credibilidad de las instituciones mediáticas. Otros resultados interesantes indican que, a diferencia de la opinión pública norteamericana, los argentinos que se ubican más a la derecha del espectro ideológico expresan mayor (y no menor) confianza, un fenómeno relacionado al bajo grado de independencia del periodismo respecto de los dueños de medios, generalmente ligados a élites sociales y sectores del establishment. Por otra parte, tal como expresa la literatura sobre el tema, los atributos individuales vinculados a la sofisticación política están negativamente relacionados con la confianza en los medios. Finalmente, temas sensibles como la inseguridad y, en especial, la corrupción, no parecen afectar demasiado los niveles individuales de credibilidad.

Los resultados de este trabajo tienen implicancias para el estudio de la política de medios. La primera atañe al rol de los medios en la gobernabilidad democrática. La literatura sobre mediatización de la política señala que, en épocas de desafección ciudadana, los actores políticos se adaptan a las lógicas mediáticas. Sin embargo, las experiencias del giro a la izquierda sugieren que la impugnación de las reglas de la política mediatizada puede ser una estrategia viable de gobernabilidad. La segunda implicancia remite al papel de la política regulatoria en el sector mediático. En tiempos de transformación de las tecnologías comunicacionales, la imagen de los medios como mediadores ante la opinión pública y como instituciones de accountability vertical está cuestionada. Esto puede habilitar a que el proceso global de innovación digital sea modulado y varíe en relación a las particularidades del proceso político.

Es deseable que futuras investigaciones atiendan aspectos no considerados en este estudio. Primero, es necesario expandir el argumento a un mayor número de países. Segundo, la literatura señala que las características propias del sistema de medios también afectan la confianza de la ciudadanía. Esto requiere examinar las propiedades del sector mediático a nivel nacional y subnacional. Tercero, es necesario adoptar un enfoque experimental para identificar los mecanismos a través de los cuales las élites moldean las percepciones acerca de los medios. Por ejemplo, sería interesante aplicar un diseño de apareamiento (matching) para determinar si los individuos "sofisticados progobierno" confían 
aún menos que los "sofisticados opositores". Cuarto, es preciso generar datos para investigar si las percepciones sobre los medios varían según la fuente del mensaje, la institución mediática y el tipo de propietario. Por último, es preciso construir indicadores para medir el efecto de la calidad de los medios sobre la credibilidad ciudadana, en lugar de recurrir a proxies como la sofisticación política. En este sentido, es recomendable observar las sensaciones sobre coberturas de escándalos y campañas, las actitudes sobre coberturas que interpretan antes que aquellas que solo reportan noticias y las percepciones explícitas sobre sesgos ideológicos de parte de los medios.

\section{REFERENCIAS}

Arpan, Laura y Arthur Raney. 2003. "An Experimental Investigation of News Source and the Hostile Media Effect." Journalism and Mass Communication Quarterly 80 (2): 265-281.

Bateson, Regina. 2010. "The Criminal Threat to Democratic Consolidation in Latin America." Ponencia presentada en el Congreso Anual de la Asociación Americana de Ciencia Política, Washington DC.

Berlo, David, James Lemert, y Robert Mertz. 1969. "Dimensions for Evaluating the Acceptability of Message Sources." Public Opinion Quarterly 33 (4): 563-576.

Booth, John, y Mitchell A. Seligson. 2009. The Legitimacy Puzzle in Latin America: Political Support and Democracy in Eight Latin American Nations. Nueva York: Cambridge University Press.

Bucy, Erik. 2003. "Media Credibility Reconsidered: Synergy Effects Between On-Air and Online News." Journalism \& Mass Communication Quarterly 80 (2): 247-264.

Bullock, John. 2011. "Elite Influence on Public Opinion in an Informed Electorate." American Political Science Review 105 (3): 496-515.

Campbell, Angus, Philip Converse, Warren Miller, y Donald Stokes. 1960. The American Voter. Chicago: University of Chicago Press.

Cappella, Joseph y Kathleen H. Jamieson. 1997. Spiral of Cynicism. Nueva York: Oxford University Press.

Carifio, James y Perla Rocco. 2007. “Ten Common Misunderstandings, Misconceptions, Persistent Myths and Urban Legends about Likert Scales and Likert Response Formats and their Antidotes." Journal of Social Sciences 3 (3): 106-116.

Carreras, Miguel. 2013. "The Impact of Criminal Violence on System Support in Latin America." Latin American Research Review 48 (3): 85-107.

Cassidy, William. 2005. "Variations on a Theme: The Professional Role Conceptions of Print and Online Newspaper Journalists." Journalism \& Mass Communication Quarterly 82 (2): 264-280.

Converse, Philip. 1964. The Nature of Belief Systems in Mass Publics. En Ideology and Discontent, editado por David E. Apter, Nueva York: The Free Press of Glencoe, 206-261.

Cook, Timothy, Paul Gronke, y John Rattliff. 2000. "Disdaining the News: American's Changing Attitudes Towards the News." Trabajo presentado en el Congreso de la Asociación Americana de Ciencia Política, Chicago, IL.

Cook, Timothy, y Paul Gronke. 2007. "The Skeptical American: Revisiting the Meanings of Trust in Government and Confidence in Institutions." Journal of Politics 67 (3): 784-803.

Craig, Stephen, Richard Niemi, y Glenn Silver. 1990. "Political Efficacy and Trust: A Report on the NES Pilot Study Items." Political Behavior 12 (3): 289-314.

D'Alessio, Dave. 2003. "An Experimental Examination of Readers' Perceptions of Media Bias." Journalism and Mass Communication Quarterly 80 (2): 282-294. 
Dalton, Russell, Paul Beck, y Robert Huckfeldt. 1998. "Partisan Cues and the Media: Information Flows in the 1992 Presidential Election." American Political Science Review 92 (1): 111-126.

Golan, Guy. 2010. "New Perspectives on Media Credibility Research." American Behavioral Scientist 54 (1): 3-7.

Gunther, Albert. 1992. "Biased Press or Biased Public? Attitudes Toward Media Coverage of Social Groups." Public Opinion Quarterly, 56 (2): 147-167

Gunther, Albert y Cindy Christen. 2002. "Projection or Persuasive Press? Contrary Effects of Personal Opinion and Perceived News Coverage on Estimates of Public Opinion." Journal of Communication 52 (1): 177-195.

Hallin, Daniel, y Paolo Mancini. 2004. Comparing Media Systems. Three Models of Media and Politics. Cambridge: Cambridge University Press.

Johnson, T. J. and Kaye, B. K. 2004. "Wag the blog: How Reliance on Traditional Media and the Internet Influence Credibility Perceptions of Weblogs Among Blog Users." Journalism and Mass Communication Quarterly 81: 622-642.

Jones, David. 2004. "Why Americans don't Trust the Media: A preliminary Analysis." International Journal of Press Politics 9 (2): 60-77.

Kitzberger, Philip. 2012. "The Media Politics of Latin America's Leftist Governments." Journal of Politics in Latin America 4 (3): 123-139.

Kitzberger, Philip. 2016. "Media Wars and the New Left: Governability and Media Democratisation in Argentina and Brazil." Journal of Latin American Studies 48 (3): 447-476.

Kitzberger, Philip. s/f. "Against the Current: The Emergence of a Media Democratization Policy Agenda in Latin America." Global Media and Communication. En prensa.

Kohring, Matthias, y Jorg Matthes. 2007. "Trust in News Media: Development and Validation of a Multidimensional Scale." Communication Research 34 (2): 231-252.

Lafferty, Barbara, y Ronald Goldsmith. 2004. "How Influential are Corporate Credibility and Endorser Attractiveness when Innovators React to Advertisements for a New High-Technology Product?" Corporate Reputation Review 7 (1): 24-36.

Layton, Matthew. 2012. "Who Trusts the Media in Latin America?" AmericasBarometer Insights Series 74. Nashville: Latin American Public Opinion Project, Vanderbilt University.

Lee, Tien-Tsu. 2010. "Why They Don't Trust the Media: An Examination of Factors Predicting Trust." American Behavioral Scientist 54 (1): 8-21.

Levitsky Steven, y Kenneth Roberts. 2011. The Resurgence of the Latin American Left. Baltimore: The Johns Hopkins University Press.

Lewis-Beck, Michael, Helmut Norpoth, William Jacoby, y Herbert Weisberg. 2008. The American Voter Revisited. Ann Arbor, MI: University of Michigan Press.

Martini, Stella. 2007. "Argentina. Prensa gráfica, delito y seguridad." En Los relatos periodísticos del crimen, ed. Germán Rey. Bogotá: Centro de Competencia en Comunicación Fundación Friedrich Ebert.

Mason, Laurie, y Clifford Nass. 1989. "How Partisan and Non-Partisan Readers Perceive Political Foes and Newspaper Bias." Journalism Quarterly 66 (3): 564-571.

Matsubayashi, Tetsuya. 2013. "Do Politicians Shape Public Opinion?" British Journal of Political Science 42 (2): 451-478.

Metzger, Miriam, Andrew Flanagin, Keren Eyal, Daisy Lemus, y Robert McCann. 2003. "Credibility in the $21^{\text {st }}$ Century: Integrating Perspectives on Source, Message, and Media Credibility in the Contemporary Media Environment." Communication Yearbook 27: 293-335.

Mochkofsky, Graciela. 2011. Pecado original. Clarín, los Kirchner y la lucha por el poder. Buenos Aires: Planeta.

Moehler, Devra y Naunihal Singh. 2011. “Whose News do You Trust? Explaining Trust in Private versus Public Media in Africa." Political Research Quarterly 64 (2): 276-292.

Newton, Kenneth y Pippa Norris. 2000. "Confidence in Public Institutions: Faith, Culture, or Performance?" En What's Troubling the Trilateral Countries, editado por Susan J. Pharr y Robert D. Putnam: Princeton: Princeton University Press, 52-73. 
Nicholson, Stephen. 2009. "Polarizing Cues." American Journal of Political Science 56 (1): 52-66. Norman, Geoff. 2010. "Likert Scales, Levels of Measurement and the "Laws" of Statistics." Advances in Health Sciences Education 15: 625-32.

Schmitt, Kathleen, Albert Gunther y Janice Liebhart. 2004. "Why Partisans See Mass Media as Biased." Communication Research 31 (6): 623-641.

Segura, Soledad, y Silvio Waisbord. 2016. Media Movements. Civil Society and Media Policy Reform in Latin America. Chicago: Chicago University Press.

Seligson, Mitchell A. 2006. "The Measurement and Impact of Corruption Victimization: Survey Evidence from Latin America." World Development 34 (2): 381-404.

Sivak, Martín. 2015. Clarín. La era Magnetto. Buenos Aires: Planeta.

Sundar, Shyam, y Clifford Nass. 2001. "Conceptualizing Sources in Online News." Journal of Communication 51 (1): 52-72.

Tsfati, Yariv, y Joseph Cappella. 2003. "Do People Watch What They do Not Trust? Exploring the Association Between News Media Skepticism and Exposure." Communication Research 30 (5): 504-529.

Tsfati, Yariv. 2003. "Media Skepticism and Climate of Opinion Perception." International Journal of Public Opinion Research 15 (6): 5-82.

Tsfati, Yariv. 2010. "Online News Exposure and Trust in the Mainstream Media. Exploring Possible Associations." American Behavioral Scientist 54 (1): 22-42.

Vallone, Robert, Lee Ross, y Mark Lepper. 1985. "The Hostile Media Phenomenon: Biased Perception and Perceptions of Media Bias in Coverage of the Beirut Masacre." Journal of Personality and Social Psychology 49 (3): 577-585.

Vincent, Lucía. 2014. “Los medios y los fines. El gobierno de Néstor Kirchner y su relación con la prensa." Tesis doctoral. Universidad Nacional de General San Martín, Argentina.

Vommaro, Gabriel e Iván Schuliaquer. 2014. "Definir la escena pública. Medios y política en las democracias sudamericanas: Argentina y Uruguay 2003-2013." Observatorio Latinoamericano 14: 139-162.

Waisbord, Silvio. 2000. "Media in South America. Between the Rock of the State and the Hard Place of the Market." En De-Westernizing Media Studies, editado por James Curran y Myung-Jin Park. Londres: Routledge, 50-62.

Waisbord, Silvio. 2013. Vox populista. Medios, periodismo, democracia. Buenos Aires: Gedisa.

Weitz-Shapiro, Rebecca. 2008. "The Local Connection: Local Government Performance and Satisfaction with Democracy in Argentina." Comparative Political Studies 41 (3): 285-308.

Zaller, John. 1992. The Nature and Origins of Mass Opinion. Cambridge: Cambridge University Press.

Germán Lodola es investigador adjunto del Consejo Nacional de Investigaciones Científicas y Técnicas (CONICET) y profesor asistente del Departamento de Ciencia Política y Estudios Internacionales de la Universidad Torcuato Di Tella. Doctor en Ciencia Política por la Universidad de Pittsburgh (2010). Correo electrónico: glodola@utdt.edu

Philip Kitzberger es investigador adjunto del Consejo Nacional de Investigaciones Científicas y Técnicas (CONICET) y profesor ordinario del Departamento de Ciencia Política y Estudios Internacionales de la Universidad Torcuato Di Tella. Doctor en Filosofía por la Universidad de Buenos Aires (2003). Correo electrónico: pkitzberger@utdt.edu 